\title{
Accounting for the Error due to Unresolved Scales in Ensemble Data Assimilation: A Comparison of Different Approaches
}

\author{
Thomas M. HAMiLL \\ University of Colorado, and NOAA-CIRES Climate Diagnostics Center, Boulder, Colorado \\ JEFFREY S. WHITAKER \\ NOAA-CIRES Climate Diagnostics Center, Boulder, Colorado
}

(Manuscript received 5 June 2004, in final form 16 March 2005)

\begin{abstract}
Insufficient model resolution is one source of model error in numerical weather predictions. Methods for parameterizing this error in ensemble data assimilations are explored here. Experiments were conducted with a two-layer primitive equation model, where the assumed true state was a T127 forecast simulation. Ensemble data assimilations were performed with the same model at T31 resolution, assimilating imperfect observations drawn from the T127 forecast. By design, the magnitude of errors due to model truncation was much larger than the error growth due to initial condition uncertainty, making this a stringent test of the ability of an ensemble-based data assimilation to deal with model error. Two general methods, "covariance inflation" and "additive error," were considered for parameterizing the model error at the resolved scales (T31 and larger) due to interaction with the unresolved scales (T32 to T127). Covariance inflation expanded the background forecast members' deviations about the ensemble mean, while additive error added specially structured noise to each ensemble member forecast before the update step.

The method of parameterizing this model error had a substantial effect on the accuracy of the ensemble data assimilation. Covariance inflation produced ensembles with analysis errors that were no lower than the analysis errors from three-dimensional variational (3D-Var) assimilation, and for the method to avoid filter divergence, the assimilations had to be periodically reseeded. Covariance inflation uniformly expanded the model spread; however, the actual growth of model errors depended on the dynamics, growing proportionally more in the midlatitudes. The inappropriately uniform inflation progressively degradated the capacity of the ensemble to span the actual forecast error.

The most accurate model-error parameterization was an additive model-error parameterization, which reduced the error difference between $3 \mathrm{D}$-Var and a near-perfect assimilation system by $\sim 40 \%$. In the lowest-error simulations, additive errors were parameterized using samples of model error from a time series of differences between T63 and T31 forecasts. Scaled samples of differences between model forecast states separated by $24 \mathrm{~h}$ were also tested as additive error parameterizations, as well as scaled samples of the T31 model state's anomaly from the T31 model climatology. The latter two methods produced analyses that were progressively less accurate. The decrease in accuracy was likely due to their inappropriately long spatial correlation length scales.
\end{abstract}

\section{Introduction}

Ensemble-based atmospheric data assimilation techniques are actively being explored as a potential replacement for or complement to three- or fourdimensional variational data assimilation (3D-Var or 4D-Var; Parrish and Derber 1992; Le Dimet and Tala-

Corresponding author address: Dr. Thomas M. Hamill, NOAACIRES CDC, R/CDC 1, 325 Broadway, Boulder, CO 80305-3328. E-mail: tom.hamill@noaa.gov grand 1986; Courtier et al. 1994; Rabier et al. 1998, 2000). Data assimilation algorithms statistically adjust prior forecasts to newly available observations to generate reduced error initial conditions suitable for numerical weather predictions. As such, these methods require error statistics of both the prior forecast (the "background") and the observations. Typically, error distributions are also assumed to be normally distributed, stationary (time invariant), and perhaps homogeneous (spatially invariant in some sense, such as along a latitude circle). Ensemble-based data assimilation 
methods relax some of these assumptions. In particular, the background-error statistics are estimated from an ensemble of forecasts and can vary in magnitude and spatial structure depending on the flow of the day. This permits them in theory to provide a more accurate adjustment of forecasts to new observations, resulting in reduced-error analyses. Ensemble-based techniques typically require an ensemble of $\sim 20$ to a few hundred members of short-range forecasts and as many parallel data assimilation updates. Hence, these methods are computationally expensive, similar to 4D-Var. For more background on ensemble-based methods see, for example, Evensen (1994), Evensen and van Leeuwen (1996), Houtekamer and Mitchell (1998), Burgers et al. (1998), Tippett et al. (2003), Anderson (2003), Evensen (2003), and Lorenc (2003).

In perfect-model tests, the ensemble filters produced simulations with dramatically lower errors than were achieved with competing methods (e.g., Hamill and Snyder 2000, 2002; Anderson 2001). Only within the last year or two have ensemble-based methods been tested in realistic numerical weather prediction models with real observations. In that case the perfect-model assumptions must be dropped, and the forecast uncertainty due to model error must be parameterized in some fashion. Etherton and Bishop (2004) compared variants of ensemble filters in the presence of model error and found hybrid methods (Hamill and Snyder 2000) generally superior. Houtekamer et al. (2005) have parameterized model deficiencies with "additive error," adding noise to each member of the ensemble of background forecasts, in their case selecting noise consistent in structure with a 3D-Var background-error covariance model. Their initial results showed that ensemble-based assimilation methods were competitive with but not superior to an operational 3D-Var. Whitaker et al. (2004, hereafter W04) tested the assimilation of a sparse network of surface pressure observations for purposes of generating a long-term, historical reanalysis. W04 found that the analysis errors from the ensemble data assimilation were significantly less than were achieved with a simple 3D-Var. With the sparse observations, the form of the background-error covariance model had more of an impact on the accuracy of the assimilations (Hamill and Snyder 2000). In W04, model errors were treated through "covariance inflation," inflating the ensemble members' spread about the ensemble mean. Despite the generally good results, their data assimilations produced too little analysis spread in data-rich regions and too much spread in data-sparse regions.

How important is the specific method of parameterizing model errors in ensemble-based data assimilation applications? This is a question we seek to answer in part in this study. Model error, of course, can be introduced from many causes, such as imperfect parameterizations (Buizza et al. 1999; Palmer 2001). In this paper, we will choose to examine only one particular aspect of model error, the errors introduced by the truncation of the forecast model and the resulting lack of interaction with the smaller scales of motion. In these simulations, the lack of interaction will be shown to result in a deficiency of spread, but will not systematically bias the ensemble very much. We will then consider whether any of several types of additive noise can be as good or better a parameterization of this model error than covariance inflation. Our simulation experiment will assume that the true state is a global simulation at triangular truncation T127, while the assimilation will be carried out at T31 resolution, assimilating imperfect observations sampled from the T127 nature run. We will test whether differences between T63 and T31 forecasts are an effective additive model error parameterization, and we will also test the effects of scaled differences between forecast model states separated by $24 \mathrm{~h}$ and scaled differences of random model states from the climatological mean state.

Because of its computational speed and general resemblance to operational numerical weather prediction (NWP) models, we will use a simple, global two-layer primitive equation (PE) model for these ensemble data assimilation experiments. We begin with an examination of the characteristics of the model itself and of short-term forecast errors due to truncation (section 2). Section 3 provides a description of the ensemble-based data assimilation methodology and various candidate techniques for parameterizing model error. Section 4 describes the experiment, section 5 examines the relative accuracy of the assimilations using various modelerror parameterizations and discusses these results, and the conclusions are presented in section 6 .

\section{Forecast model}

\section{a. Model design}

In this paper, results will be based on experiments with a dry, global, two-layer PE model. The forecast model was described in Zou et al. (1993, see their appendix A) and was used in Hamill et al. (2001) for ensemble data assimilation experiments in a perfectmodel context. The model is spectral and was run at three triangular truncations: T127, T63, and T31. The model state vector consists of vorticity and divergence spectral coefficients at two levels as well as coefficients of layer thickness $\Delta \pi$ for each layer, where $\pi$ is the Exner function. There is a simple, zonal wavenumber-2 
terrain with a maximum amplitude of $2000 \mathrm{~m}$ at $45^{\circ} \mathrm{N}-\mathrm{S}$ and $45^{\circ}$ and $135^{\circ} \mathrm{E}$, smoothly tapering to $0 \mathrm{~m}$ at the Poles and equator (minimum amplitudes of $-2000 \mathrm{~m}$ are located at $45^{\circ} \mathrm{N}-\mathrm{S}$ and $45^{\circ} \mathrm{W}$ and $135^{\circ} \mathrm{E}$ ). The model is forced by Newtonian relaxation to a prescribed interface Exner function with a damping time scale of $\tau_{\text {diab }}=20$ days. A fourth-order Runge-Kutta scheme is used for the numerical integration. The time steps are 5, 10, and $20 \mathrm{~min}$ for the T127, T63, and T31 resolutions. There is $\nabla^{8}=\left(\nabla^{2}\right)^{4}$ hyperdiffusion with a 6 -h $e$-folding time scale for the shortest resolvable scale. The diffusion acting on a given wavenumber will therefore increase as the truncation is made more severe. Other parameters are the same as in Zou et al. (1993), with the exception that the upper-layer potential temperature is specified to be $\theta_{2}=310 \mathrm{~K}, \theta_{1}=280 \mathrm{~K}$, the model top $z_{\text {top }}=1.5 \times 10^{4} \mathrm{~m}$, and the lower-layer drag coefficient $\tau_{\text {drag }}=4$ days. The Exner function at the model top $\pi_{\text {top }}=c_{p}-\left(g z_{\text {top }} / \theta_{1}\right)$ is fixed and set to a value of $\sim 478.5 \mathrm{~J} \mathrm{~kg}^{-1} \mathrm{~K}^{-1}$. The surface Exner function is diagnosed from $\pi_{\text {top }}+\Delta \pi_{2}+\Delta \pi_{1}$.

In the experiments that follow, the model is initialized from a state of rest plus a barotropic vorticity perturbation and allowed to spin up for 100 days before beginning the forecast and assimilation experiments.

\section{b. Model-and truncation-error characteristics}

Error-doubling times, as computed from the leading Lyapunov vectors (Legras and Vautard 1996), are shorter when the model is run at higher resolution. The error doubling times are 3.78 days at T31, 2.16 days at T63, and 1.88 days at T127. As in Snyder and Hamill (2003), the leading Lyapunov vectors are closely related to the jet stream dynamics (not shown).

For all resolutions, the model exhibits upper-level westerly jets in the midlatitudes and an easterly jet in the Tropics (Fig. 1a). The tropical easterly jet is less pronounced at T127 resolution while the midlatitude westerly jets are more pronounced. Subtropical lowerlevel easterly jets are slightly less pronounced at T127 (Fig. 1b). The midlatitudinal gradient of the upperlayer thickness $\Delta \pi$ (Fig. 1c) is slightly larger in magnitude in the T127 simulation, consistent with the stronger midlatitude jet.

Figure 2 shows the kinetic energy power spectra from nature runs at T127, T63, and T31 resolutions as a function of total wavenumber $k$. The spectral slope at subsynoptic scales is shallower than the $k^{-3}$ expected with two-dimensional turbulence but steeper than the $k^{-5 / 3}$ of $3 \mathrm{D}$ turbulence. At wavenumbers around 30, the power in the T31 nature run is damped relative to the two higher-resolution simulations, a consequence of the (a) Upper-level Zonal Wind $(\mathrm{m} / \mathrm{s})$

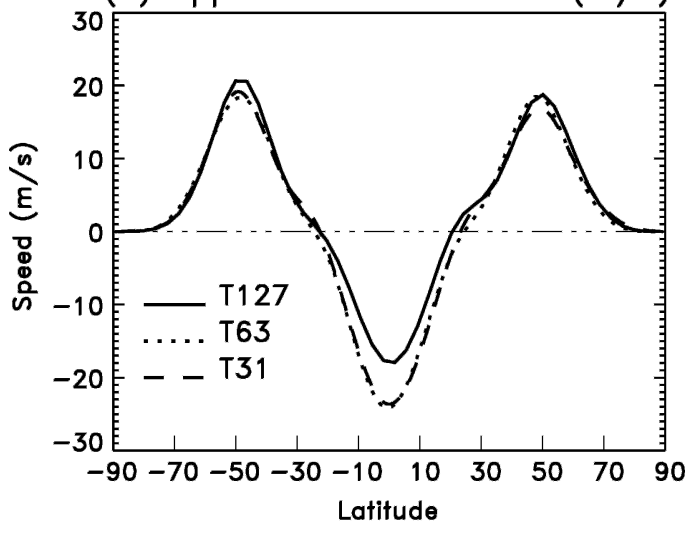

(b) Lower-level Zonal Wind $(\mathrm{m} / \mathrm{s})$

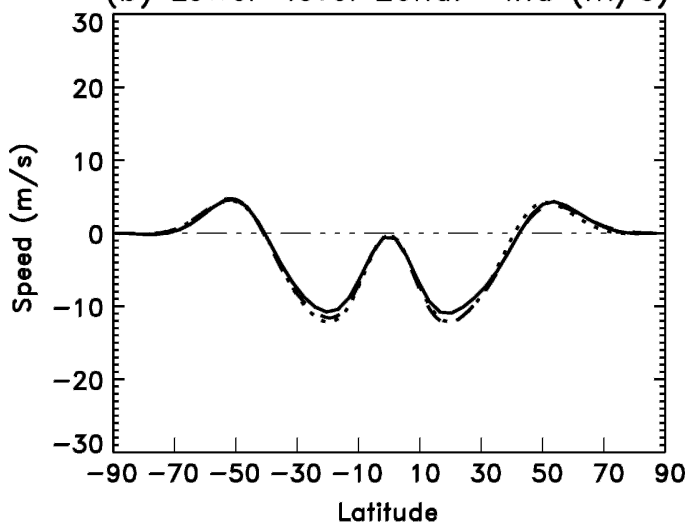

(c) Upper-level $\Delta \pi$

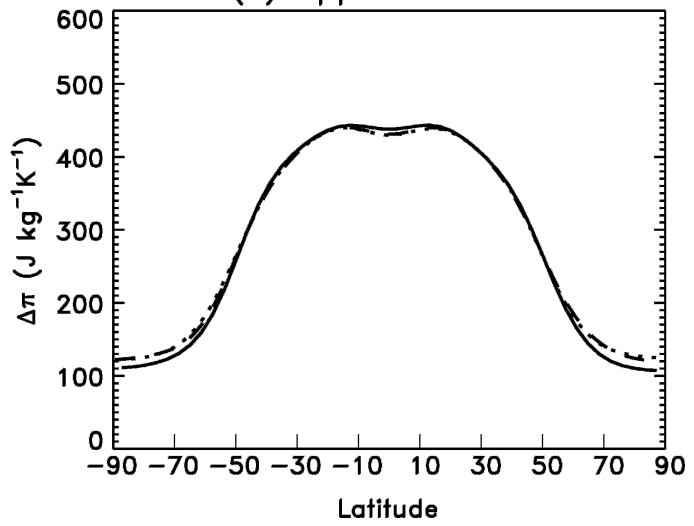

FIG. 1. Zonal mean statistics for nature runs at T127, T63, and T31 resolutions. (a) Upper-layer zonal wind, (b) lower-layer zonal wind, and (c) upper-layer $\Delta \pi$.

$\nabla^{8}$ hyperdiffusion, which selectively damps the shortest retained scales.

For subsequent experiments, let us assume that our forecast system is only able to resolve scales T31 and larger, hereafter called the "resolved scales." The errors in the resolved scales due to interaction with the scales of motion smaller than T31, the "unresolved 


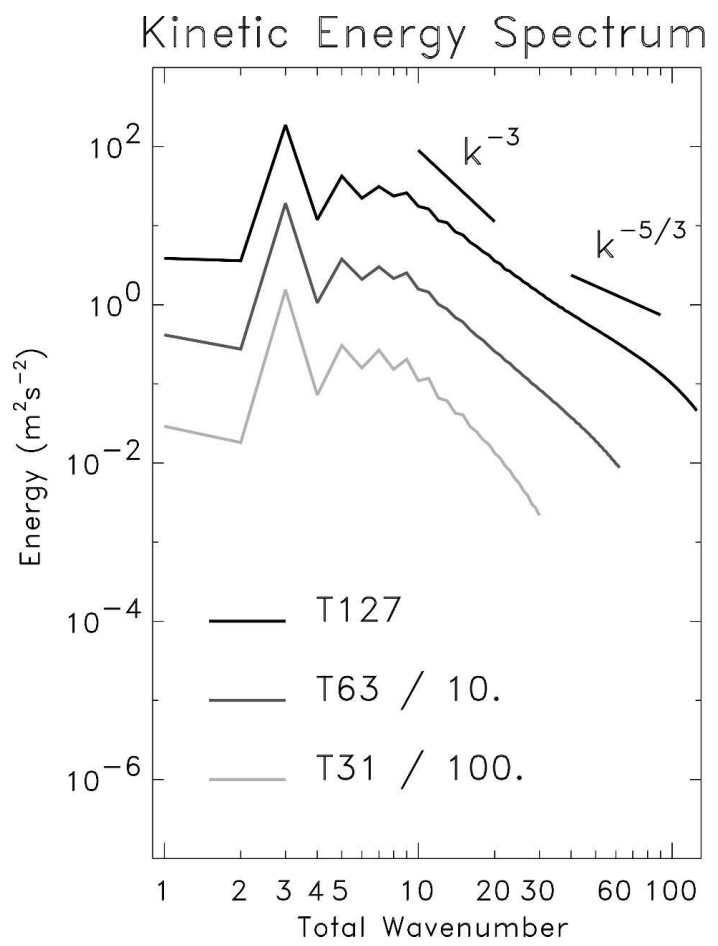

FIG. 2. Kinetic energy spectrum of nature runs at T127, T63, and T31 resolutions. Curves for T63 and T31 are shifted by one and two orders of magnitude, respectively.

scales," is what constitutes the model error in these simulations. We will assume that the "real" atmosphere evolves according to the forecast dynamics described by the same model, but at T127 resolution. Short-term model error is diagnosed as follows: first, let $\mathbf{x}^{127}(t)$ denote the true model state at time $t$ at full T127 resolution. Let $\mathcal{M}^{127}\{\cdot\}$ denote the forecast model operator at T127 resolution taking the state forward one time unit: $\mathbf{x}^{127}(t+1)=\mathscr{M}^{127}\left\{\mathbf{x}^{127}(t)\right\}$. Let $\mathcal{T}[\cdot]$ denote the truncation operation of the model state to the resolved scales. Then short-term model error is thus $\mathcal{M}^{\beta 1}\left\{\mathcal{T}\left[\mathbf{x}^{127}(t)\right]\right\}-$ $\mathcal{T}\left[\mathbf{x}^{127}(t+1)\right]$. That is, the model error consists of the difference of a low-resolution forecast from a truncated initial condition minus the truncated forecast from a high-resolution model using a high-resolution initial condition. This describes the short-term forecast error in the resolved scales due to the lack of modeling of interaction with the unresolved scales. Similar frameworks for the diagnosis of model errors were proposed in Cohn and Dee [1988, their Eq. (2.6c)], Dee [1995, his Eq. (5)], and Mitchell and Daley (1997, their section 2).

Figure 3 shows a time series of model errors computed independently every $24 \mathrm{~h}$ over five consecutive time periods. At the beginning of each 24 -h period, the T31 simulation was reinitialized with the truncated T127 nature run's state, and model error was computed (a) Upper-Level T31 True $\Delta \pi$ and 24-h Model Error at $0 \mathrm{~h}$

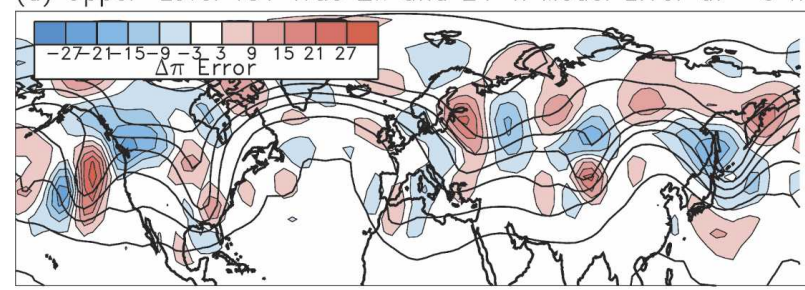

(b) Upper-Level T31 True $\Delta \pi$ and 24-h Model Error at $24 \mathrm{~h}$

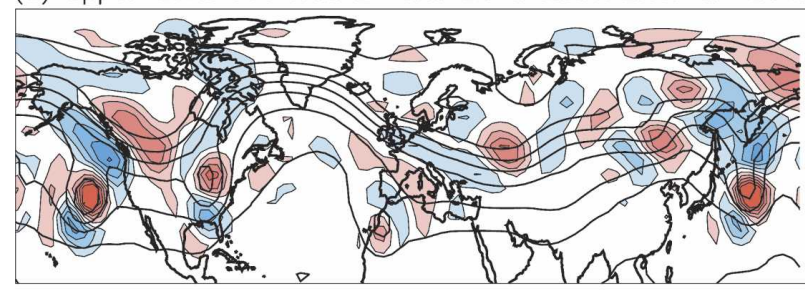

(c) Upper-Level T31 True $\Delta \pi$ and 24-h Model Error at $48 \mathrm{~h}$

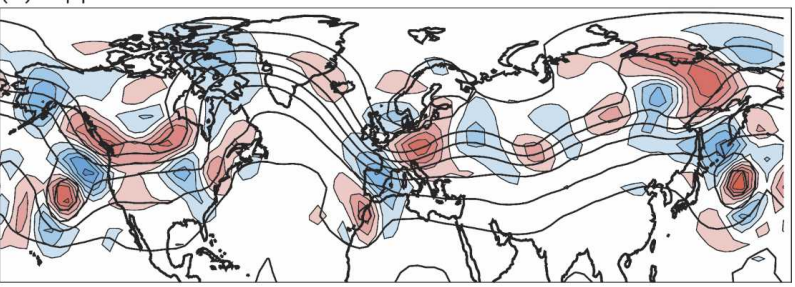

(d) Upper-Level T31 True $\Delta \pi$ and 24-h Model Error at $72 \mathrm{~h}$

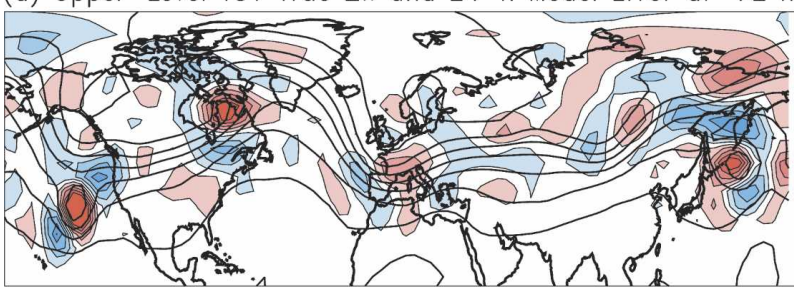

(e) Upper-Level T31 True $\Delta \pi$ and 24-h Model Error at $96 \mathrm{~h}$

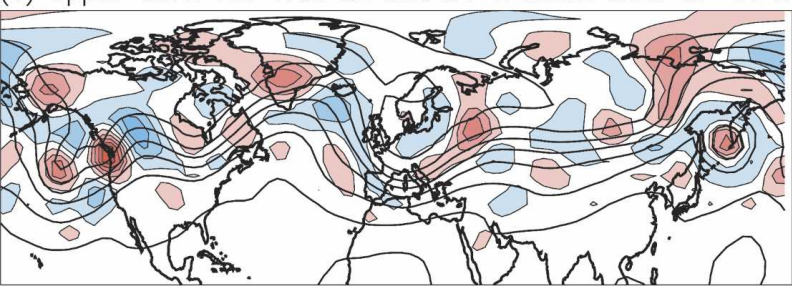

FIG. 3. Time series of upper-layer thickness 24-h model errors. True state of upper-layer $\Delta \pi$ resolved scales overplotted in solid lines (contour interval $50 \mathrm{~J} \mathrm{~kg}^{-1} \mathrm{~K}^{-1}$ ). Geographical features are overlaid only for perspective.

at the end of the next 24-h period. The model error appears to have some temporally correlated features associated with certain shortwaves. There was also a substantial amount of error that appeared to be more random (an examination showed that a small amount of gravity wave noise was introduced by the truncation, which is mostly damped after $24 \mathrm{~h}$ ). Perturbations were generally larger in the regions of large gradients of $\Delta \pi$, typically in the midlatitudes. 
Model errors were initially small in scale. As the time span of the forecast over which model errors are computed was increased, the model errors grew in size and scale (Fig. 4), peaking eventually at the synoptic scales. After an initial transient period, these results took on many of the characteristics of the predominantly baroclinic growth witnessed in Tribbia and Baumhefner (2004) rather than the classic upscale growth associated with a $k^{-3}$ spectrum discussed in Lorenz (1969) and Leith and Kraichnan (1972). However, the errors here do not accumulate as quickly at the planetary scales as in the Tribbia-Baumhefner study.

Figure 4 also shows model-error spectra when the model was truncated at T63 resolution instead of T31. The overall T63 24-h model-error spectrum was approximately an order of magnitude smaller, since far more of the power of the overall spectrum was resolved. The peak power was at a slightly shorter wavenumber, and the errors grew faster, so the T63 model errors were a greater fraction of the T31 model error at $120 \mathrm{~h}$ than at $3 \mathrm{~h}$. Errors at the smallest scales of the T63 simulation appear to be supersaturated at $3 \mathrm{~h}$ (a variance higher than climatological variance) but were subsequently damped by the diffusion. Overall, the amplitude of model error due to truncation is decreased as the resolution is increased. See also Mitchell and Daley (1997) for further discussion of errors due to unrepresented scales.

\section{Ensemble data assimilation methodology}

The general ensemble-based assimilation methodology proceeds as follows. First, assume that a set of $n$ perturbed initial conditions is available that presumably samples from the distribution of background (first guess) errors. Perform $n+1$ parallel data assimilation updates using an ensemble-based assimilation algorithm, updating the ensemble-mean and $n$ perturbations from the mean to the newly available observations, modeling the background-error covariances using the ensemble. Next, make $n$ forecasts forward to the next data assimilation time. This step may include adjusting these forecast ensemble members in some manner to account for model errors. Repeat the update and forecast steps. Below, we consider the details of the update and forecast steps.

\section{a. Updating with the ensemble square root filter}

The assimilation scheme used here has been named the ensemble square root filter (EnSRF). A complete description of it and the rationale for its use is provided in Whitaker and Hamill (2002). The underlying prin-
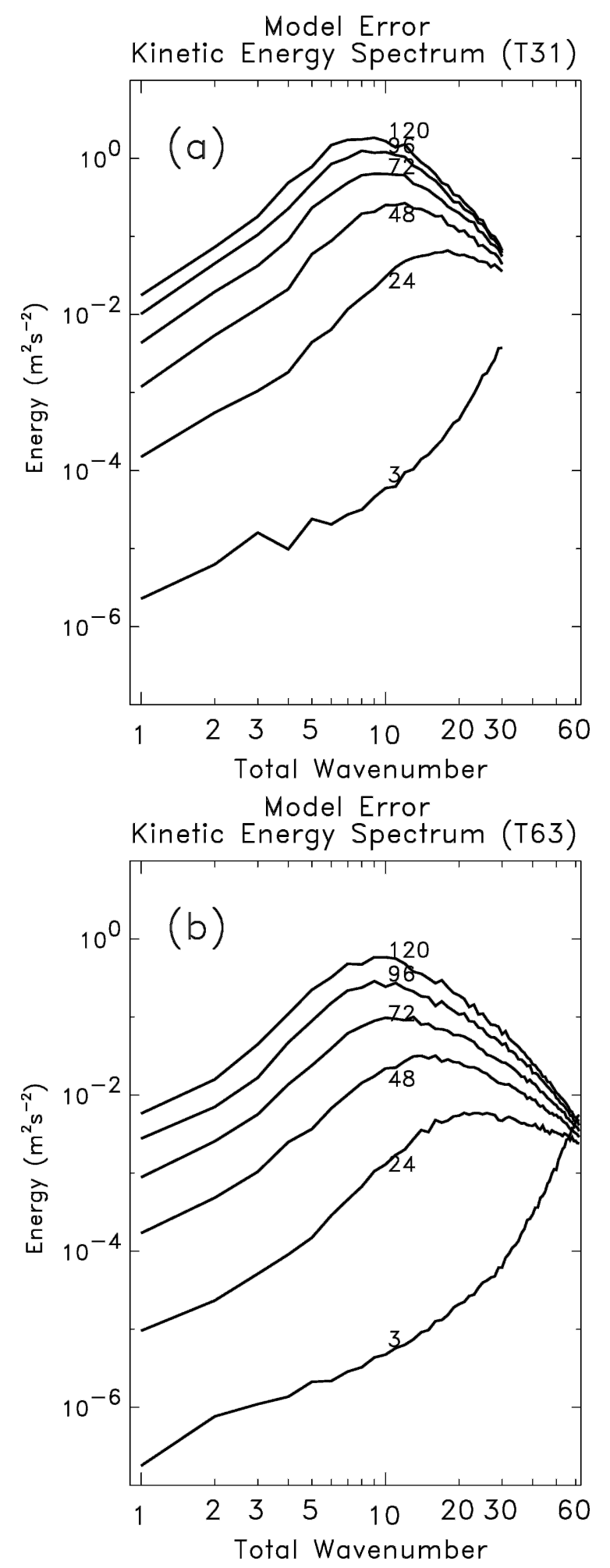

FIG. 4. Kinetic energy spectrum of model errors due to lack of interactions with unresolved scales, accumulated at 3, 24, 48, 72, 96, and 120 h. (a) Spectrum of model errors when model is truncated at (a) T31 and (b) T63. 
ciple is to run an ensemble of parallel forecast and data assimilation cycles, ensuring that the ensemble-mean analysis and the analysis-error covariance as estimated by the ensemble are consistent with those predicted by Kalman filter theory.

Let $\mathbf{y}^{\circ}(t)$ be a set of observations at time $t$ and $\mathbf{H}$ be a linear operator that converts the model state to the observation space. Let $\mathbf{X}^{\mathrm{b}}(t)=\left[\mathbf{x}_{1}^{\mathrm{b}}(t), \ldots, \mathbf{x}_{n}^{\mathrm{b}}(t)\right]$ be a matrix whose column vectors are the ensemble of $n$ forecasts, and similarly, let $\overline{\mathbf{X}^{\mathrm{b}}}(t)=\left[\overline{\mathbf{x}^{\mathrm{b}}}(t), \ldots, \overline{\mathbf{x}^{\mathrm{b}}}(t)\right]$ denote an matrix where each column vector is the ensemble-mean forecast: $\overline{\mathbf{x}^{\mathrm{b}}}(t)=(1 / n) \sum_{i=1}^{n} \mathbf{x}_{i}^{\mathrm{b}}(t)$. Let $\mathbf{P}^{\mathrm{b}}(t)$ be the background-error covariance matrix approximated using the sample covariance from an ensemble of model forecasts; $\mathbf{P}^{\mathrm{b}}(t)=[1 /(n-1)]\left[\mathbf{X}^{\mathrm{b}}(t)-\overline{\mathbf{X}^{\mathrm{b}}}(t)\right]$ $\left[\mathbf{X}^{\mathrm{b}}(t)-\overline{\mathbf{X}^{\mathrm{b}}}(t)\right]^{\mathrm{T}}$ [note that $\mathbf{P}^{\mathrm{b}}(t)$ is never explicitly formed, only the matrices $\mathbf{P}^{\mathrm{b}}(t) \mathbf{H}^{\mathrm{T}}$ and $\mathbf{H} \mathbf{P}^{\mathrm{b}}(t) \mathbf{H}^{\mathrm{T}}$ are formed; see Houtekamer and Mitchell 1998, their Eqs. (13) and (15)]. Also let $\mathbf{R}$ be the observation-error covariance matrix.

Following Whitaker and Hamill (2002), it is convenient in the EnSRF to update the equations for the ensemble mean (denoted by an overbar) and each member's deviation from the mean (prime) separately:

$$
\begin{aligned}
\overline{\mathbf{x}}^{\mathrm{a}}(t) & =\overline{\mathbf{x}}^{\mathrm{b}}(t)+\mathbf{K}\left[\mathbf{y}^{\circ}(t)-\mathbf{H} \overline{\mathbf{x}}^{\mathrm{b}}(t)\right] \\
\mathbf{x}_{i}^{\prime \mathrm{a}}(t) & =(\mathbf{I}-\tilde{\mathbf{K}} \mathbf{H}) \mathbf{x}_{i}^{\prime}{ }^{\mathrm{b}}(t)
\end{aligned}
$$

Here, the superscript a denotes the analysis, $\mathbf{K}$ is the traditional Kalman gain,

$$
\mathbf{K}=\mathbf{P}^{\mathrm{b}}(t) \mathbf{H}^{\mathrm{T}}\left[\mathbf{H} \mathbf{P}^{\mathrm{b}}(t) \mathbf{H}^{\mathrm{T}}+\mathbf{R}\right]^{-1},
$$

and $\tilde{\mathbf{K}}$ is a the "reduced" gain used to update deviations from the ensemble mean. When sequentially processing independent observations, $\mathbf{K}, \tilde{\mathbf{K}}, \mathbf{H} \mathbf{P}^{\mathrm{b}}(t)$ and $\mathbf{P}^{\mathrm{b}}(t) \mathbf{H}^{\mathrm{T}}$ are all vectors with the same number of elements as the model-state vector, and $\mathbf{H} \mathbf{P}^{\mathrm{b}}(t) \mathbf{H}^{\mathrm{T}}$ and $\mathbf{R}$ are scalars. Thus, as first noted by J. Potter in 1964 (Maybeck 1979):

$$
\tilde{\mathbf{K}}=\left[1+\sqrt{\frac{\mathbf{R}}{\mathbf{H P}^{\mathrm{b}}(t) \mathbf{H}^{\mathrm{T}}+\mathbf{R}}}\right]^{-\mathbf{1}} \mathbf{K} .
$$

The quantity multiplying $\mathbf{K}$ in Eq. (4) is a scalar between 0 and 1 . This means that, in order to obtain the analysis-error covariance consistent with the Kalman filter, one updates deviations from the ensemble mean using a modified Kalman gain that is reduced in magnitude relative to the traditional Kalman gain. Deviations from the mean are thus reduced less in the analysis using $\tilde{\mathbf{K}}$ than they would be using $\mathbf{K}$. For more details, see Whitaker and Hamill (2002) and Lawson and Hansen (2004).

To improve the modeling of background-error cova- riances, the covariances estimated from the ensemble are subject to modification by "localization" (e.g., Houtekamer and Mitchell 2001; Hamill et al. 2001; Mitchell et al. 2002; Lorenc 2003). The Kalman gain in (3) is replaced by a modified gain

$$
\mathbf{K}=\left[\rho_{\mathrm{S}} \circ \mathbf{P}^{\mathrm{b}}(t)\right] \mathbf{H}^{\mathrm{T}}\left\{\mathbf{H}\left[\rho_{\mathrm{S}} \circ \mathbf{P}^{\mathrm{b}}(t)\right] \mathbf{H}^{\mathrm{T}}+\mathbf{R}\right\}^{-1},
$$

where the operation $\rho_{\mathrm{S}} \circ$ in (5) denotes a Schur product (an element-by-element multiplication) of a correlation matrix $\rho_{\mathrm{S}}$ having local support with the covariance model generated by the ensemble. Again, $\left[\rho_{\mathrm{S}} \circ \mathbf{P}^{\mathrm{b}}(t)\right]$ is never explicitly formed [see Houtekamer and Mitchell (2001), their Eqs. (5) and (6)].

\section{b. Generating background forecasts}

The other necessary part of the data assimilation cycle is the propagation of the forecast ensemble forward in time with the full, nonlinear forecast model to the time of the next new observations. This ensemble is used to estimate the mean state and background-error covariances. Unfortunately, the forecast model is not perfect, so even if one of the ensemble members happened to have a perfect initial condition, its subsequent forecast would contain errors. In Kalman filters (e.g., Gelb 1974; Maybeck 1979) the background-error covariances are specified by propagating the analysis-error covariances forward using the linear tangent $\mathbf{M}$ and its adjoint of the fully nonlinear forecast model operator $\mathcal{M}$, with an addition of covariance $\mathbf{Q}$ to account for model error:

$$
\mathbf{P}^{\mathrm{b}}(t+1)=\mathbf{M} \mathbf{P}^{\mathrm{a}}(t) \mathbf{M}^{\mathrm{T}}+\mathbf{Q} .
$$

Here $\mathbf{Q}$ is assumed to be Gaussian, composed of errors $\eta \sim(0, \mathbf{Q})$ that are uncorrelated in time and uncorrelated with internal error.

The EnSRF and ensemble Kalman filter (EnKF; Burgers et al. 1998) produce covariances similar to those of the Kalman filter, using the ensemble of fully nonlinear forecasts to estimate the background-error covariances. Let $\mathbf{X}^{\mathrm{a}}(t)=\left[\mathbf{X}_{1}^{\mathrm{a}}(t), \ldots, \mathbf{X}_{\mathrm{n}}^{\mathrm{a}}(t)\right]$ be an ensemble of analyses at time $t$. Let $\mathcal{M}$ denote the forward model operator between time $t$ and $t+1$, and let $\overline{\mathcal{M} \mathbf{X}^{\mathrm{a}}}(t)$ denote an array composed of column vectors of ensemble-mean forecasts started from time $t$, that is, $\overline{\mathcal{M} \mathbf{X}^{\mathrm{a}}}(t)=\left[\overline{\mathcal{M} \mathbf{x}^{\mathrm{a}}}(t), \ldots, \overline{\mathcal{M} \mathbf{x}^{\mathrm{a}}}(t)\right]$, where $\overline{\mathcal{M} \mathbf{x}^{\mathrm{a}}}(t)=(1 / n)$ $\sum_{i=1}^{n} \mathcal{M} \mathbf{x}_{i}^{\mathrm{a}}(t)=(1 / n) \sum_{i=1}^{n} \mathbf{x}_{i}^{\mathrm{b}}(t+1)$. Then if forecast errors evolve linearly and the size $n$ of the ensemble increases, $\left[\mathcal{M} \mathbf{X}^{\mathrm{a}}(t)-\overline{\mathcal{M} \mathbf{X}^{\mathrm{a}}}(t)\right]\left[\mathcal{M} \mathbf{X}^{\mathrm{a}}(t)-\overline{\mathcal{M} \mathbf{X}^{\mathrm{a}}}(t)\right]^{\mathrm{T}}=$

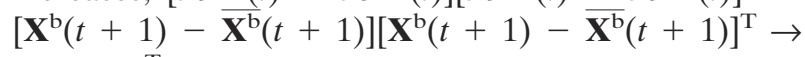
$\mathbf{M P}^{\mathrm{a}}(t) \mathbf{M}^{\mathrm{T}}$. This indicates that the ensemble can be propagated forward and used to estimate the background-error covariances, minus the missing $\mathbf{Q}$ term in 
(6). If the forward propagator introduces systematic errors, the background forecasts should be adjusted (see Dee and Todling 2000). In the present experiment, the systematic error was found to be negligible. The subsequent experiments will demonstrate the impact of parameterizing $\mathbf{Q}$ through two general techniques: covariance inflation and/or additive errors.

With covariance inflation, ensemble members' deviations about their mean are inflated by an amount $r$, slightly greater than 1.0, before the first observation is assimilated:

$$
\mathbf{x}_{i}^{\mathrm{b}}(t+1) \leftarrow r\left[\mathbf{x}_{i}^{\mathrm{b}}(t+1)-\overline{\mathbf{x}}^{\mathrm{b}}(t+1)\right]+\overline{\mathbf{x}}^{\mathrm{b}}(t+1) .
$$

Here, the operation $\leftarrow$ denotes a replacement of the previous value of $\mathbf{x}_{i}^{\mathrm{b}}(t+1)$. Application of a moderate inflation factor has been found to improve the accuracy of assimilations in perfect-model experiments (Hamill et al. 2001; Whitaker and Hamill 2002) and real-data simulations (W04). Note that inflation increases the spread of the ensemble, but it does not change the subspace spanned by the ensemble. The common assumption in the Kalman filter derivation is that model error and internal error growth are uncorrelated. If indeed the model-error projects into a substantially different subspace than the ensemble, this parameterization may not be effective. However, if the dynamically relevant part of model error is the part that projects onto the growing modes spanned by the ensemble, covariance inflation may be effective. Another issue with covariance inflation is that a single inflation factor $r$ may not be optimal over all parts of a model domain. Consider, for example, a situation where observations are very plentiful in one hemisphere and nearly nonexistent in the other. In the data-sparse hemisphere, observations will not reduce the spread of the ensemble as much during the updates, and covariance inflation may lead to an unbounded growth of ensemble variance.

Additive errors (Mitchell et al. 2002; Houtekamer et al. 2005) may avoid some of these problems. Noise $\eta_{i}$ with the same dimension as the model state is added to each ensemble member background forecast before the update cycle starts:

$$
\mathbf{x}_{i}^{\mathrm{b}}(t+1) \leftarrow \mathbf{x}_{i}^{\mathrm{b}}(t+1)+\eta_{i} .
$$

Here $\eta_{i}$ ought to sample the probability distribution of accumulated model errors $\mathbf{Q}$, that is, $\left\langle\eta_{i} \eta_{i}^{\mathrm{T}}\right\rangle=\mathbf{Q}$ (here $\langle\cdot\rangle$ denotes the expected value). Also, $\left\langle\mathbf{x}_{i}^{\prime \mathrm{b}}(t+1) \eta_{i}^{\mathrm{T}}\right\rangle=$ 0 ; that is, the model error ought to be independent of the internal error, though the model error might still be dependent on the model state. Unlike covariance inflation, the resulting modified ensemble may well span a somewhat different subspace than the unmodified en- semble. Furthermore, additive error will not arbitrarily double its effect with double the spread, as will covariance inflation.

In the subsequent section we describe tests of ensemble assimilations with additive errors generated using three different methods. The first approach was to sample differences between the resolved scales of model forecasts at different resolutions. Recall that in our experiment, the true model state was obtained from a T127 simulation and the ensemble forecasts were conducted at T31. Suppose generating a T127 simulation is a computational impossibility, but we have the ability to generate T63 simulations. The difference of the resolved scales between T63 and T31 forecasts can then be computed: $\mathcal{M}^{31}\left\{\mathcal{T}\left[\mathbf{x}^{63}(t)\right]\right\}-\mathcal{T}\left[\mathbf{x}^{63}(t+1)\right]$. This time series can be randomly sampled, and a different sample $\eta_{i}$ can be added to each ensemble member according to (8). In fact, what typically was added was $s \eta_{i}$, where $s$ represented a scaling factor greater than 1.0. That is, the additive error samples were inflated in size somewhat before being added to the background forecasts.

Another ad hoc approach that was tested was to add scaled-down differences between random model states from the forecast model climatology, that is, $\eta_{i}=$ $s\left[\mathbf{x}^{31}\left(t_{r}\right)-\overline{\mathbf{x}}^{31}\right]$, where $t_{r}$ is a random time from the time series of the T31 nature run, $\overline{\mathbf{x}}^{31}$ is the mean climatological state, and again $s$ is a scaling factor. A similar approach was used for generating ensemble perturbations by Schubert and Suarez (1989). A third additive error approach used scaled-down short-term $(24 \mathrm{~h})$ forecast tendencies: $\eta_{i}=s\left[\mathbf{x}^{31}\left(t_{r}\right)-\mathbf{x}^{31}\left(t_{r}-24 h\right)\right]$, where again $t_{r}$ is a random time from the time series.

Figure 5 shows the spatially lagged correlations of additive errors for upper-layer thickness generated through these three processes, as well as the lagged correlation of the true model errors, that is, $\mathcal{M}^{31}\left\{\mathcal{T}\left[\mathbf{x}^{127}\right.\right.$ $(t)]\}-\mathcal{T}\left[\mathbf{x}^{127}(t+1)\right]$. Lower-layer thickness errors had similar characteristics. For both Tropics and extratropics, the differences between model forecasts at T63 and T31 resolutions produced additive errors with the shortest correlation length scales, very similar to the correlation length scale of the actual model error. The length scales from 24-h forecast tendencies were substantially longer, and samples from climatology had the longest length scales, being especially unrealistic in the Tropics. Figure 5 also shows the correlation structure of typical background forecasts from one of the subsequent ensemble data assimilation experiments (experiment 5, described in section $4 \mathrm{~b}$ ). Additive errors from differences between T63 and T31 forecasts shortened the correlation length scale of the background errors, while climatological additive noise lengthened it. 

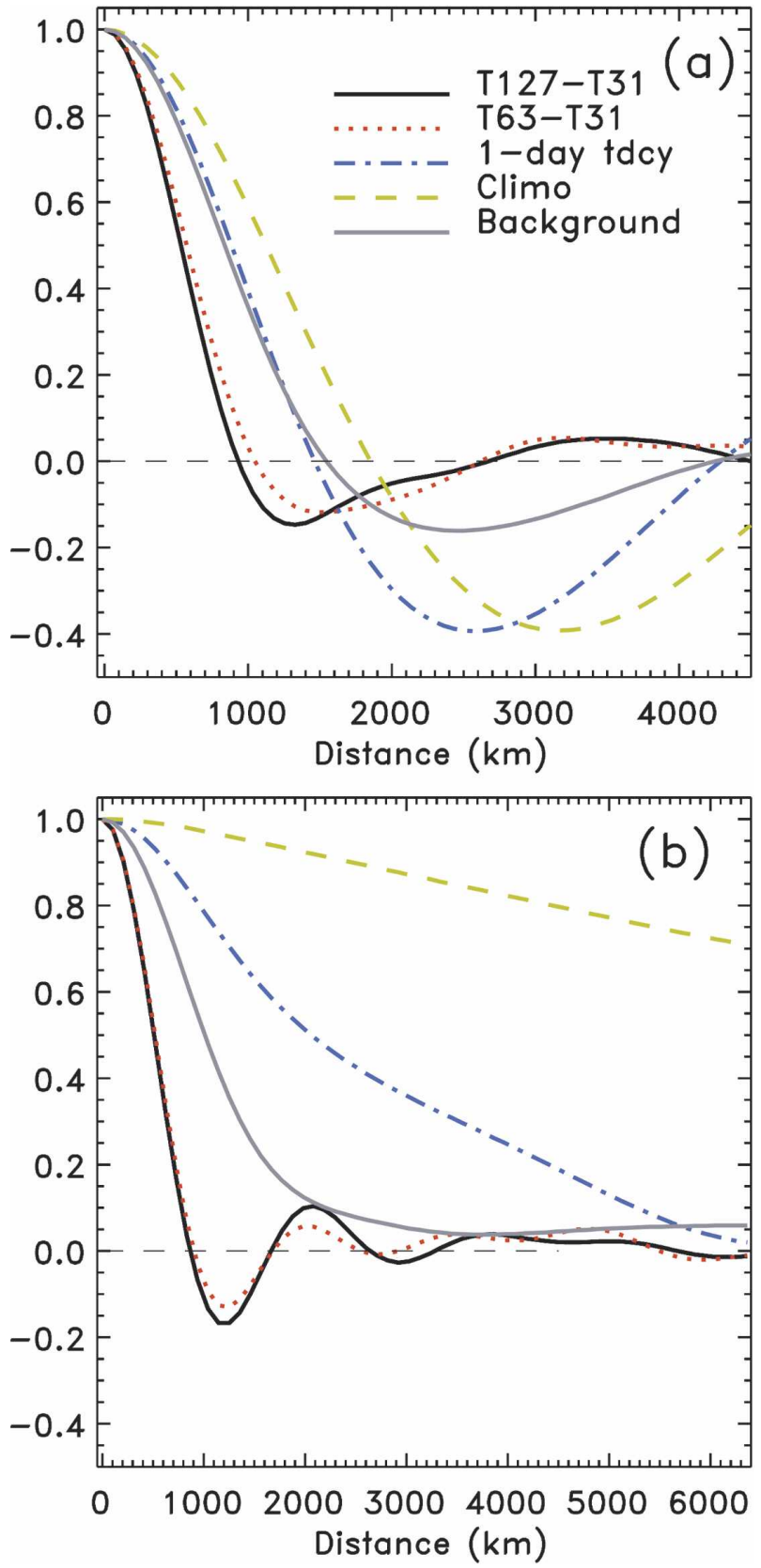

FIG. 5. Spatially lagged correlation along (a) $45^{\circ} \mathrm{N}$ latitude and (b) equator of $\Delta \pi$ as a function of zonal distance for various additive error models, as well as the correlation of the background forecast ensemble.

\section{Experiment design}

a. Observations, ensemble configuration, and evaluation technique

We now describe a large number of data assimilation experiments. In each experiment, observations of $\Delta \pi_{2}$ were taken at a set of nearly equally spaced locations on a spherical geodesic grid (Fig. 6). The observations consisted of the T127 true state plus errors drawn from a distribution with zero mean and standard deviation of $8.75 \mathrm{~J} \mathrm{~kg}^{-1} \mathrm{~K}^{-1}$. Observation errors were independent at each location and time, and observations were assimilated every $24 \mathrm{~h}$.

In all the experiments, the ensemble size was 208 members. The ensemble was initialized with random draws from the climatology of the assimilating model. The data assimilation proceeded over a 150-day period; the first 50 days were discarded as a spinup period, and error statistics were accumulated over the remaining 100 days. Aside from experiment 1, a perfect-model simulation at T127, all the other experiments were conducted at T31 resolution.

After some preliminary experimentation, the chosen covariance localization for all experiments used the Gaspari and Cohn (1999) compactly supported fifthorder piecewise rational function that decayed to zero at $5000 \mathrm{~km}$. This length scale was chosen after trying several cutoff distances in perfect-model experiments with the T127 model (experiment 1 below).

The ensemble-mean analysis error and spread (standard deviation of the ensemble about its mean) were measured in three norms, a globally averaged, massweighted kinetic energy norm, an upper-layer thickness norm (since the model top is fixed, this is equivalent to a measurement of interface Exner function), and sur-

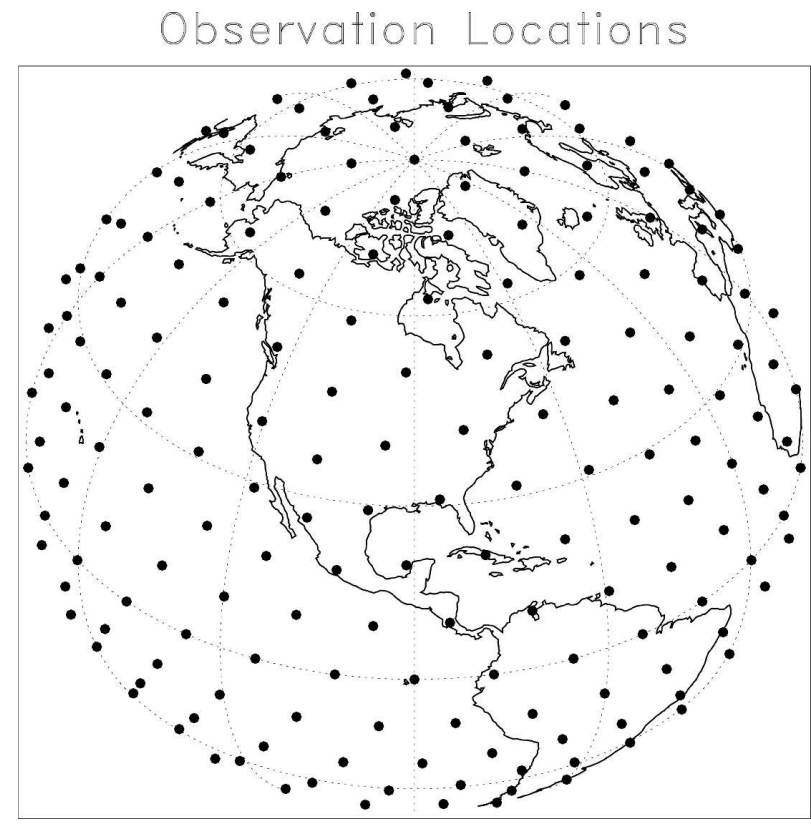

FIG. 6. Observation locations. Observations were generated globally on a spherical geodesic grid. There are 362 observation locations worldwide. 


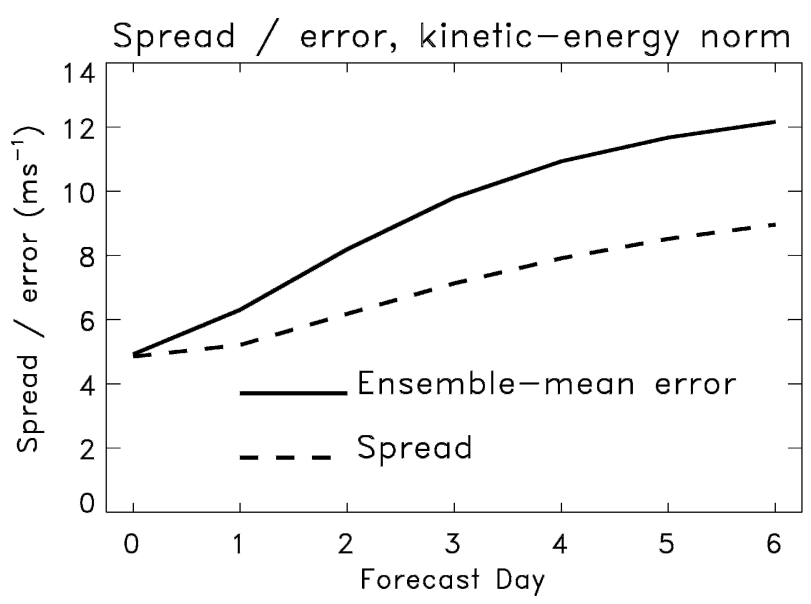

FIG. 7. Growth of ensemble spread and ensemble-mean error in the kinetic energy norm, taken from the perfect additive error experiment (No. 4).

face Exner function norm. The kinetic energy norm at a given time is

$$
\|\cdot\|_{\mathrm{ke}}=\left[\frac{\int_{S} \int_{L} \pi\left(\mathbf{u}^{\prime 2}+\mathbf{v}^{\prime 2}\right) d S d L}{\iint_{S} \int_{L} \pi d S d L}\right]^{0.5} .
$$

Here, $\mathbf{u}^{\prime}$ and $\mathbf{v}^{\prime}$ denote the model wind components' ensemble-mean error or spread, $\pi$ again refers to the model state's Exner function, $S$ refers to the integration over the sphere, and $L$ is the integration over the model layers. The interface height norm is

$$
\|\cdot\|_{\Delta \pi_{2}}=\frac{1}{A}\left(\int_{S} \Delta \pi_{2}^{\prime 2} d S\right)^{0.5},
$$

where $\Delta \pi_{2}^{\prime}$ denotes the upper-layer Exner function thickness ensemble-mean error or deviation from the mean, and A is the surface area of the earth. The surface Exner function norm is

$$
\|\cdot\|_{\Delta \pi_{1+2}}=\frac{1}{A}\left[\int_{S}\left(\Delta \pi_{1}^{\prime}+\Delta \pi_{2}^{\prime}\right)^{2} d S\right]^{0.5},
$$

where $\Delta \pi_{1}^{\prime}$ is the lower-layer Exner function thickness ensemble-mean error or perturbation.

Model error is a very prominent component of the total error in these experiments, arguably more than it is in operational ensemble forecasting (e.g., Simmons and Hollingsworth 2002, their Fig. 6). Figure 7 examines the 100-day-average growth of ensemble forecast spread compared to the ensemble-mean error, taken from experiment 4, described below. Compared with the figure from Simmons and Hollingsworth, it is apparent that the growth of spread lags far behind the ensemble-mean error, significantly more than it does in a recent version of the European Centre for MediumRange Weather Forecasts (ECMWF) model.

\section{b. Experiments}

What now follows is a description of each data assimilation experiment, summarized in Table 1.

Experiment 1, T127 perfect model, performed the ensemble data assimilations using the EnSRF in a perfectmodel context, assimilating the T127 observations using an ensemble of forecasts at T127 with $r=1.01$ (some covariance inflation has been found to be helpful in perfect-model simulations; see Hamill et al. 2001; Whitaker and Hamill 2002). To be consistent with the rest of the data assimilation experiments conducted at T31 resolution, the error and spread statistics were evaluated only for scales T31 and larger.

Experiment 2, covariance inflation, used an EnSRF algorithm with the model error parameterized with a straightforward covariance inflation. Various values of

TABlE 1. Table of data assimilation experiments performed. The first column denotes the experiment number. The second column the name of the experiment. The third column denotes the general data assimilation methodology. The fourth column indicates the

\begin{tabular}{|c|c|c|c|c|c|c|}
\hline Expt No. & Name & Method & $r$ & $s$ & Additive error type & Bias correction? \\
\hline 1 & T127 perfect model & EnSRF & 1.01 & $\mathrm{n} / \mathrm{a}$ & $\mathrm{n} / \mathrm{a}$ & $\mathrm{n} / \mathrm{a}$ \\
\hline 2 & Covariance inflation & EnSRF & 1.08 & $\mathrm{n} / \mathrm{a}$ & $\mathrm{n} / \mathrm{a}$ & $\mathrm{n} / \mathrm{a}$ \\
\hline 3 & Restarted cov inflation & EnSRF & 1.10 & $\mathrm{n} / \mathrm{a}$ & $\mathrm{n} / \mathrm{a}$ & $\mathrm{n} / \mathrm{a}$ \\
\hline 4 & T127 additive error & EnSRF & $\mathrm{n} / \mathrm{a}$ & 1.00 & $\mathrm{~T} 127$ & Yes \\
\hline 5 & T63 additive & EnSRF & $\mathrm{n} / \mathrm{a}$ & 1.20 & $\mathrm{~T} 63$ & Yes \\
\hline 6 & T63 analogs & EnSRF & $\mathrm{n} / \mathrm{a}$ & 1.20 & T63 global analogs & Yes \\
\hline 7 & 24-h tendency & EnSRF & $\mathrm{n} / \mathrm{a}$ & 0.25 & 24-h tendency & No \\
\hline 8 & Climatology & EnSRF & $\mathrm{n} / \mathrm{a}$ & 0.25 & Climatology & No \\
\hline 9 & 3D-Var & 3D-Var & $\mathrm{n} / \mathrm{a}$ & 1.40 & $\mathrm{n} / \mathrm{a}$ & $\mathrm{n} / \mathrm{a}$ \\
\hline
\end{tabular}
amount of covariance inflation, $r$. The fifth column indicates the amount of rescaling of additive error, $s$. The sixth column indicates the additive error type. The seventh column indicates whether additive errors in EnSRF assimilations were subjected to a bias correction. 
TABLE 2. Global ensemble-mean analysis error (Err) and ensemble spread (Spr), measured in kinetic energy norm $\left(\mathrm{ke}_{\mathrm{e}}, \mathrm{units} \mathrm{m} \mathrm{s}^{-1}\right)$, upper-layer Exner function thickness norm $\left({ }_{\Delta \pi_{2}}\right.$, units $\left.\mathrm{J} \mathrm{kg}^{-1} \mathrm{~K}^{-1}\right)$, and surface Exner function norm $\left({ }_{\Delta \pi_{1+2}}, \mathrm{units} \mathrm{J} \mathrm{kg}^{-1} \mathrm{~K}^{-1}\right)$. Numbers in parentheses indicate the percentage improvement between experiment 1, T127 perfect model, and experiment 9, 3D-Var.

\begin{tabular}{|c|c|c|c|c|c|c|}
\hline Expt No. & $\mathrm{Err}_{\mathrm{ke}}$ & $\mathrm{Spr}_{\mathrm{ke}}$ & $\operatorname{Err}_{\Delta \pi_{2}}$ & $\operatorname{Spr}_{\Delta \pi_{2}}$ & $\operatorname{Err}_{\Delta \pi_{1+2}}$ & $\operatorname{Spr}_{\Delta \pi_{1+2}}$ \\
\hline 1 & 3.67 & 3.96 & 5.37 & 5.51 & 1.17 & 0.97 \\
\hline 2 & - & - & (Diverged) & - & - & - \\
\hline 3 & $5.62(7)$ & 5.47 & $7.74(15)$ & 5.04 & $1.83(-18)$ & 2.31 \\
\hline 4 & $4.92(40)$ & 4.85 & $7.10(38)$ & 6.81 & $1.25(86)$ & 0.86 \\
\hline 5 & $4.93(40)$ & 4.81 & $7.14(37)$ & 6.62 & $1.28(80)$ & 0.82 \\
\hline 6 & $4.96(38)$ & 4.76 & $7.10(38)$ & 6.52 & $1.27(82)$ & 0.82 \\
\hline 7 & $5.08(33)$ & 4.18 & $7.21(34)$ & 5.53 & $1.45(50)$ & 1.14 \\
\hline 8 & $5.56(10)$ & 5.36 & $7.30(31)$ & 5.89 & $1.98(-44)$ & 1.93 \\
\hline 9 & 5.76 & $\mathrm{n} / \mathrm{a}$ & 8.16 & $\mathrm{n} / \mathrm{a}$ & 1.73 & $\mathrm{n} / \mathrm{a}$ \\
\hline
\end{tabular}

covariance inflation were tried, but while these methods performed well initially, all simulations had the tendency over many weeks to become numerically unstable. For subsequent discussion, assume the covariance inflation is $r=1.08$, that is, perturbations were inflated by $8 \%$ at the beginning of each assimilation cycle.

Experiment 3, restarted covariance inflation, was a modification of experiment 2. Since covariance inflation algorithms typically outperformed 3D-Var experiments (described later) for a short period of time at the beginning of the assimilation, we considered an experiment where the EnSRF was rerun in overlapping 20day windows (doubling the cost of the assimilations). The first 10 days in each window were discarded as a spinup period, and statistics were accumulated for the remaining 10 days. Thus, the 100 days over which statistics were accumulated represented 10 overlapping data assimilation experiments. In this experiment, $r=1.10$.

Experiment 4, T127 additive error, used additive errors and no covariance inflation. In this case, additive errors for each member were randomly drawn from a time series of the true additive errors, found by comparing the T127 and T31 simulations using the procedure described in section $2 \mathrm{~b}$. This simulation was used as a basis for comparing how skillful other additive error parameterizations were.

Experiment 5, T63 additive, used only additive errors randomly drawn from differences between T63 and T31 simulations (section $2 \mathrm{~b}$ ). The scaling factor $s$ for inflating additive model-error samples was set to 1.20 , chosen by experimentation.

Experiment 6, T63 analogs, set $s=1.20$ as in experiment 5 , but in this experiment nonrandom times were selected from the time series of differences between T63 and T31 simulations. At each data assimilation time, we searched a 10 000-day time series of initial conditions from the T31 simulation and found the 208 times in that time series that were the closest fit to the observations at this time. The analogs were a somewhat better fit to the observations, typically around $20 \%-$ $40 \%$ closer fit than the random model states. Since model error appeared to be highly state dependent (Fig. 3), the hypothesis was that selecting additive model-error samples from relatively similar states would provide a better parameterization than from random states.

Experiment 7, 24-h tendency, additive errors were randomly drawn from a sample of differences between T31 nature run model states separated by $24 \mathrm{~h}$. These additive errors were scaled by 0.25 , a constant found through experimentation to produce the lowest errors.

Experiment 8, climatology, additive errors were randomly drawn from a sample of anomalies from the model's climatological mean state, scaled by 0.25 , chosen through experimentation.

Experiment 9, 3D-Var, was an experiment simulating a three-dimensional variational analysis by updating a single model state using a static background-error covariance estimate. The static background-error covariances were formed from an ensemble consisting of 208 random samples of actual background error from experiment 5 inflated by $40 \%$, chosen through experimentation. The same $5000-\mathrm{km}$ localization of covariances was used. A similar approach to simulating 3DVar in an ensemble filter framework was described in Zhang and Anderson (2003) and Evensen (2003).

\section{Results}

Table 2 summarizes the time-averaged ensemblemean error and spread of the analyses. For reference, the errors of climatology were approximately $28 \mathrm{~m} \mathrm{~s}^{-1}$ in the kinetic energy norm. The accuracy of the resolved scales from experiment 1, the T127 perfect model, produced the lowest error in all norms. Experiment 2, a straightforward application of covariance in- 

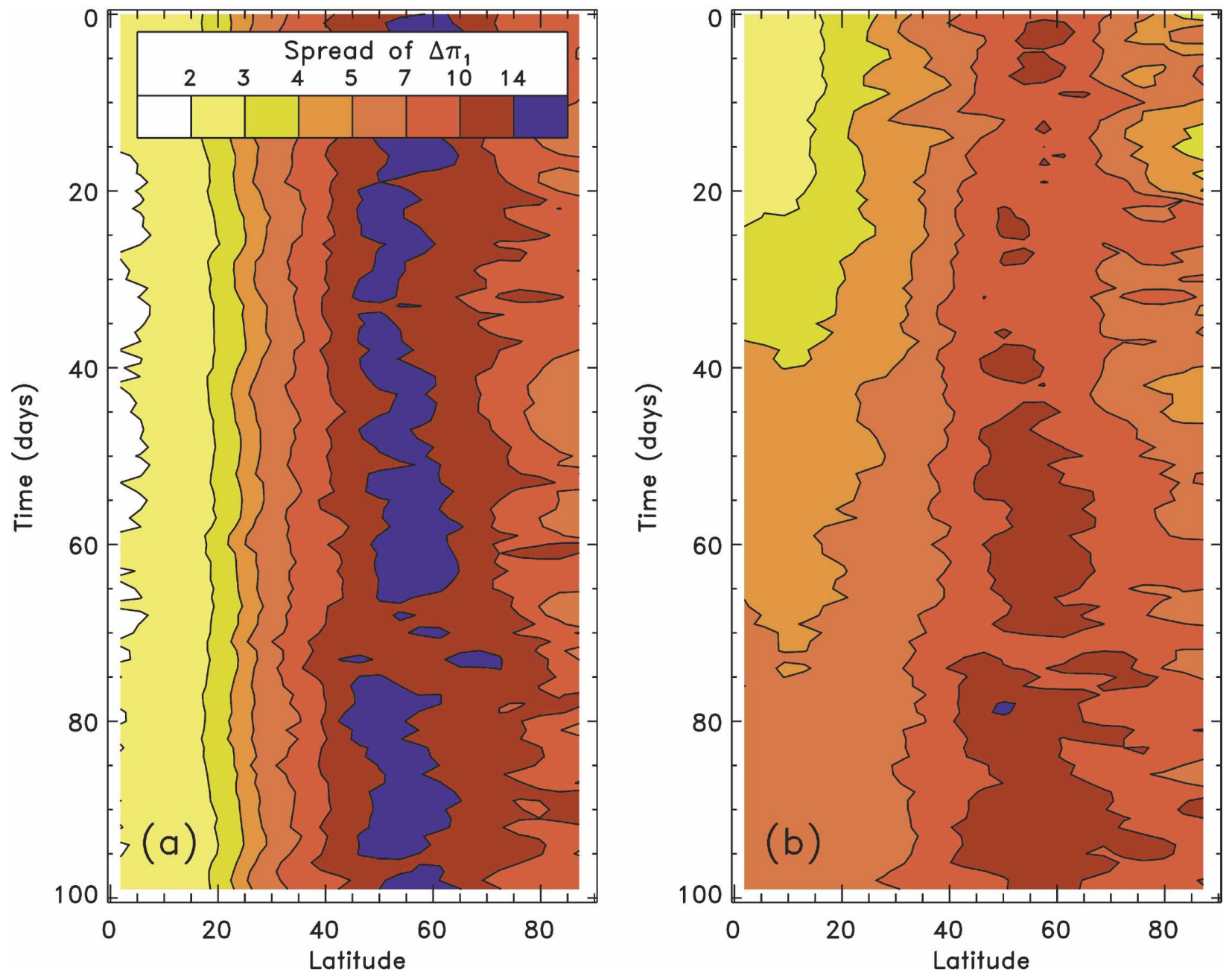

FIG. 8. Hovmöller diagrams of zonally averaged background spread of $\Delta \pi_{1}$ from the (a) T63 additive experiment, and (b) covariance inflation experiment, here initialized with the background ensemble from the T63 additive experiment.

flation, became numerically unstable. However, if the ensemble was regularly restarted, as it was in experiment 3 , then the assimilations produced analyses with $\sim 7 \%, 15 \%$, and $-18 \%$ reduction in error, measured as the fractional difference in error between 3D-Var (experiment 9) and the T127 perfect model (experiment 1) using the three norms in Eqs. (9)-(11).

The T127 additive error, the T63 additive and T63 analogs experiments all had nearly the same errors. These produced $\sim 40 \%, 38 \%$, and $86 \%$ relative reductions in error for the T63 additive experiment. Interestingly, the parameterization using the T63 runs were equally skillful in using T127 samples of the additive error (experiment 4), indicating that the use of model differences from T63 simulations was a highly effective choice of parameterization. The use of closer analogs of model error (experiment 6) did not have a noticeable impact on assimilation accuracy.
Why was the covariance inflation experiment substantially less accurate than these additive error experiments? Fig. 8a shows that the zonally averaged background spread in the T63 additive simulation reached a quasi-equilibrium state, decreased by the update but growing by a similar amount during the forecast and from the addition of additive noise. In the covariance inflation simulation, there was a continual growth of spread in the Tropics (Fig. 8b). Why? Figure 9 shows the zonal and time average of $\Delta \pi_{1}$ spread for the background ensemble and for the model errors due to truncation. Each profile was normalized by its maximum zonal value, shown in parentheses. The relative proportion of model error to background error at the equator is approximately half its proportion in the midlatitudes. Hence, a covariance inflation tuned to the midlatitudes will introduce twice as much model error at the equator as is appropriate during each assimilation cycle. 


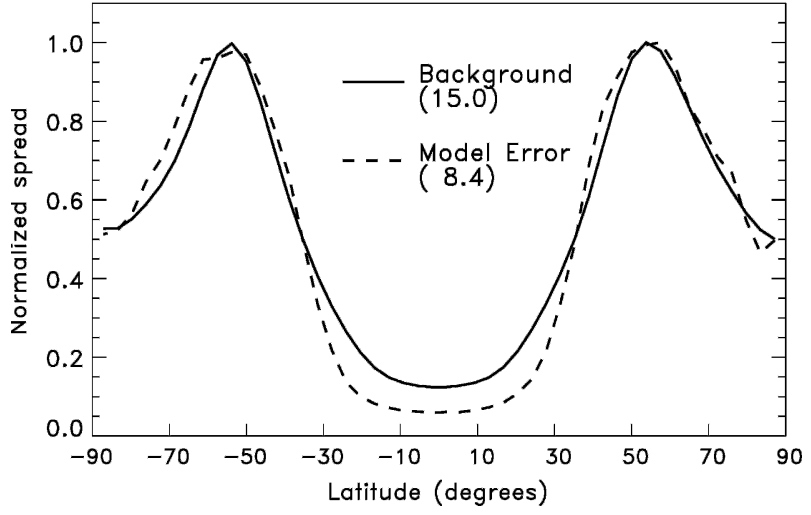

FIG. 9. Zonal- and time-averaged latitudinal profiles of background spread and model error of $\Delta \pi_{1}$, normalized by the largest average value. Magnitude of these values are in parentheses.

We can follow how the background-error covariance model grows increasingly unrealistic in the covariance inflation assimilation experiment. In a stable data assimilation cycle such as the T63 additive error simula- tion, the increments to layer thicknesses $\Delta \pi_{1}$ and $\Delta \pi_{2}$ were strongly anticorrelated in this model (Fig. 10). Hence, when an observation of $\Delta \pi_{2}$ made a large increment to $\Delta \pi_{2}$ at nearby grid points, it typically made a large correction of similar magnitude but opposite sign to $\Delta \pi_{1}$, so the overall increment to the surface Exner function $\pi_{\text {top }}+\Delta \pi_{1}+\Delta \pi_{2}$ was much smaller $\left(\pi_{t o p}\right.$ is a constant). With uniform covariance inflation, model errors were repeatedly overestimated in the Tropics. Since analysis increments were related to the background spread at the observation location, this implied that due to the growing tropical spreads, tropical observations then began to introduce larger and larger adjustments to the background. Increasingly, these increments became less realistic. In this particular model, for instance, the unrealistic increments can be seen by examining the increments to surface Exner function from the observations of $\Delta \pi_{2}$. In the T63 additive simulation, the adjustments remained small and stable over time (Fig. 11a). In the covariance inflation simulation, the ensemble lost the strong anticorre-

(a) Ensemble Mean Background $\Delta \pi_{2}$ and $K\left(\Delta \pi_{2}\right)$ at 10 days, T63 Additive

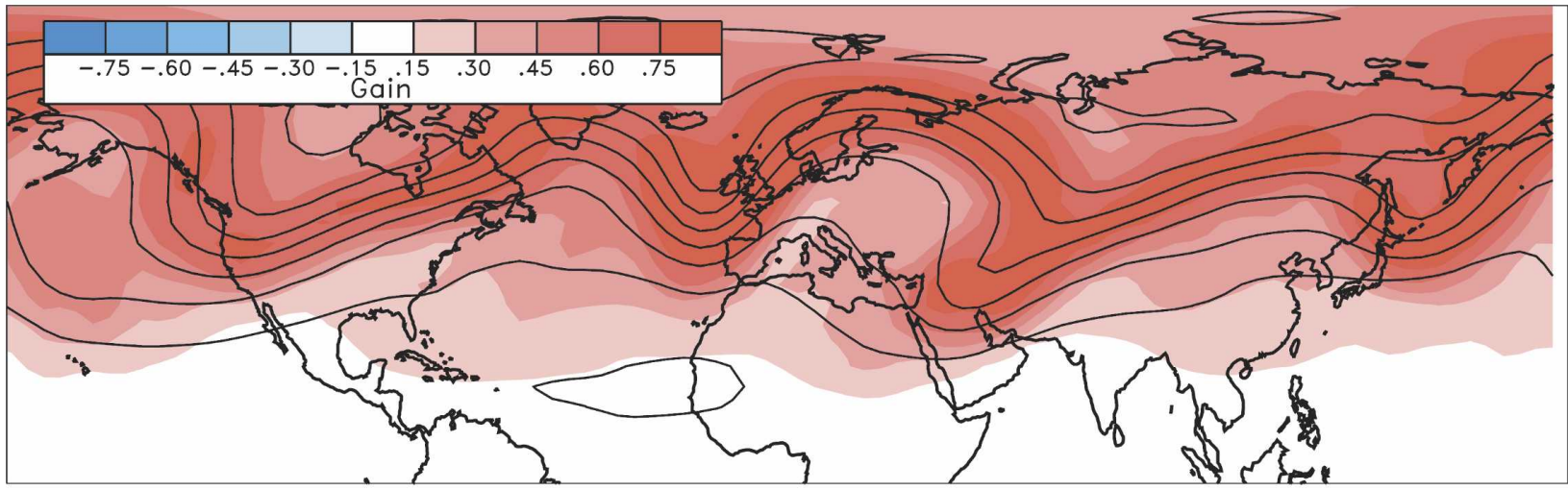

(b) Ensemble Mean Background $\Delta \pi_{2}$ and $K\left(\Delta \pi_{1}\right)$ at 10 days, T63 Additive

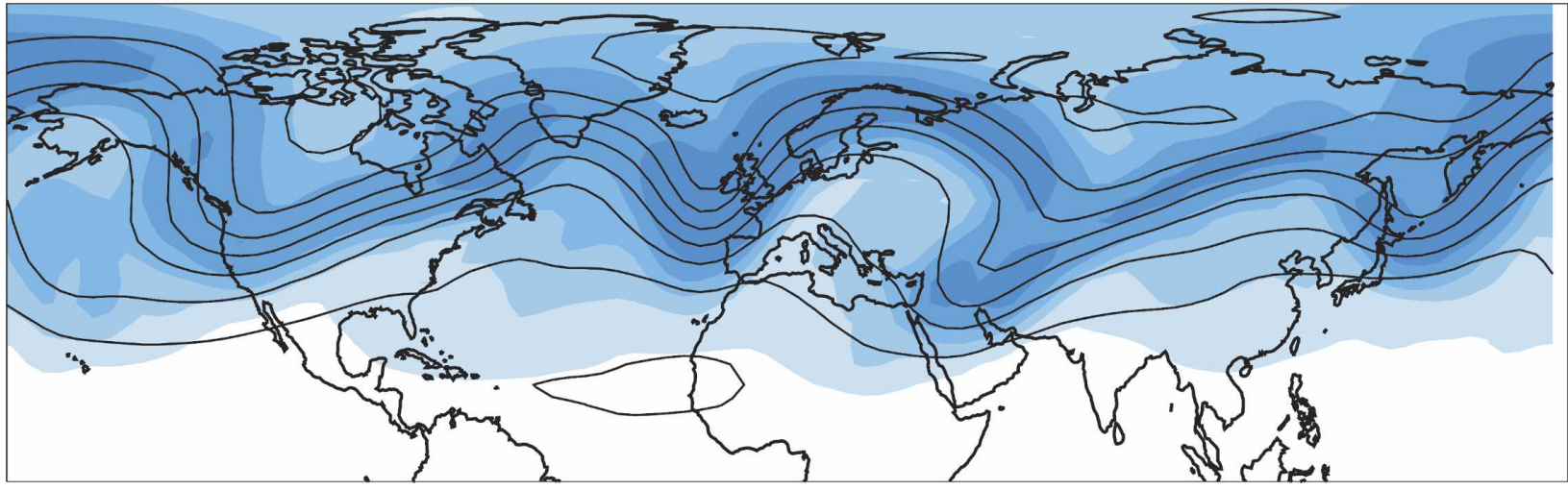

FIG. 10. Upper-layer ensemble-mean background $\Delta \pi_{2}$ (solid, contours every $50 \mathrm{~J} \mathrm{~kg}^{-1} \mathrm{~K}^{-1}$ ) and the Kalman gain (colors) for (a) the upper-layer $\Delta \pi_{2}$ and (b) the adjacent lower-layer $\Delta \pi_{1}$ at time $t=10$ days from the T63 additive experiment. 
(a) Zonal avg. of $\left|\mathrm{K}\left(\Delta \pi_{1}+\Delta \pi_{2}\right)\right|$ T63 Additive

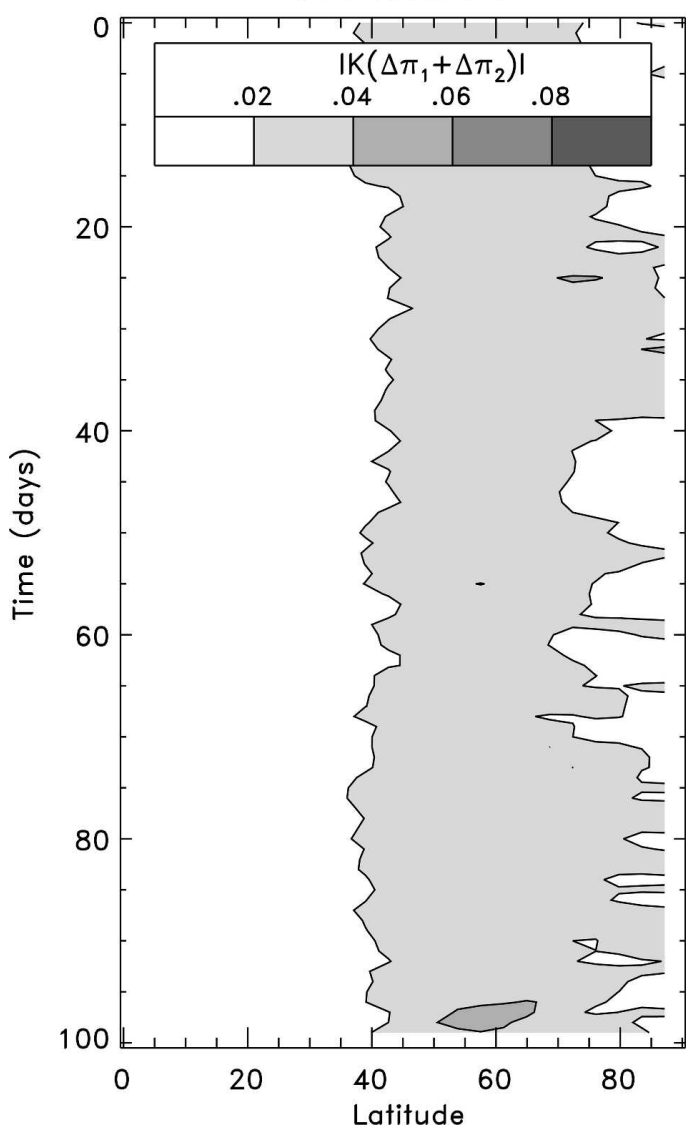

(b) Zonal avg. of $\left|\mathrm{K}\left(\Delta \pi_{1}+\Delta \pi_{2}\right)\right|$ Covariance Inflate

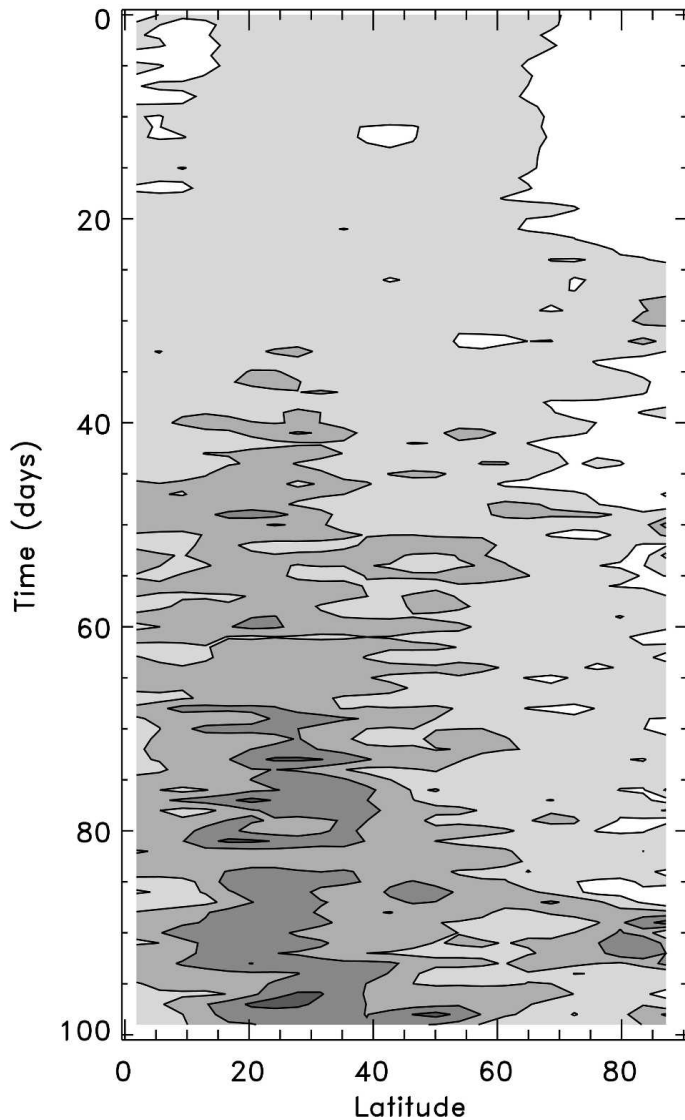

FIG. 11. Hovmöller diagrams of zonally averaged Kalman gain $\mathbf{K}$ of changes to surface Exner function $\Delta \pi_{1}+$ $\Delta \pi_{2}$ from the (a) T63 additive and (b) covariance inflation experiments.

lation between layer thicknesses, and unrealistically large adjustments to total column thickness resulted (Fig. 11b).

This behavior of the ensemble filter was somewhat different than the classical "filter divergence" problem. Here, the problem was not that the background-error covariances were systematically underestimated so that the observations were effectively ignored. Rather, errors increased because the background-error covariance model became corrupted. We conclude that a uniform, large covariance inflation factor to parameterize model error is likely to cause data assimilation problems if the model error does not grow in proportion to the background spread, as covariance inflation assumes [see also the discussion of inflation problems for convective-scale data assimilation in Snyder and Zhang (2003) their section 6b].

Perhaps a more accurate result could have been obtained had we tested more complicated inflation approaches. For example, perhaps a location-dependent inflation factor, akin to the "mask" used in the breeding technique of Toth and Kalnay (1997), could ameliorate some of these problems, as might the approach of Zhang et al. [2004, see their Eq. (5)]. Conceptually, it is of course desirable to limit the number of tunable parameters in the data assimilation algorithm.

We next consider whether forecast accuracy varied with the specific type of additive model-error parameterization. Parameterizing the model error with $24-\mathrm{h}$ tendencies and climatology produced progressively less accurate assimilations. The 24-h tendency additive errors produced analyses that were $33 \%, 34 \%$, and $50 \%$ relative improvements, while additive errors sampled from climatology produced assimilations that were $10 \%$ and $31 \%$ better and $44 \%$ worse in the three norms (recall that the T63 additive experiment produced $41 \%$, $38 \%$, and $85 \%$ reductions). Examining the analysis error characteristics in the Tropics versus the extratropics (not shown), the increased error for the climatology additive error experiment was most pronounced in the 
Tropics, where the additive errors had their most unrealistic covariance structure (Fig. 5).

Synthesizing these results, misspecification of the spatial structure of additive errors degraded the ensemble data assimilation analysis accuracy. Why? The true model errors in these simulations were relatively small in scale. When the additive errors samples were inappropriately large in scale, they modified the background ensemble so its perturbations were also too large in scale. When assimilating a single observation, the analysis increment is proportional to the background error covariance between a grid point and the observation location. Consequently, an overly large spatial scale of background-error covariances will cause the observations to have an inappropriately large influence in adjusting the analysis at distances far from the observation location, thus, decreasing analysis accuracy.

A characteristic seen in nearly all the ensemble simulations in Table 2 is that the spread for the surface Exner function is much less than the ensemble-mean error, even in the perfect-model experiment. The surface Exner function is not actually a state variable, but is diagnosed from the sum of layer thicknesses. We suspect that the larger deficiency in spread is due to larger errors in these diagnosed cross covariances of the state variables, for the spread deficiency was noted even in the T127 perfect-model simulation.

\section{Discussion and conclusions}

In these experiments, we considered different methods for parameterizing the model error in ensemble assimilations due to truncation of the model resolution. Of course, model errors in full numerical weather prediction models can be caused by many other factors, such as improper parameterizations of other subgridscale processes such as convection not included in this simplified model. Our choice here was to try to understand one relatively simple source of error and explore which methods were most effective for treating it. The experimental setup was designed to produce large model errors, probably a larger contribution to forecast error than occurs in current-generation numerical models.

The experiment was conducted with a two-layer primitive equation model. The true state was a T127 forecast nature run. Ensemble data assimilations were performed with the same model at T31 resolution, assimilating imperfect observations drawn from the T127 forecast. Several methods were considered for parameterizing the model error at the resolved scales (T31 and larger) due to interaction with the unresolved scales (T32 to T127). "Covariance inflation" simply inflated the background forecast members' deviations about the ensemble mean. Another, "additive errors," added specially structured noise to each ensemble member.

The method of parameterizing this model error had a substantial effect on the accuracy of ensemble data assimilations. The best additive model-error parameterization was able to reduce the relative error between $3 \mathrm{D}-$ Var and a near-perfect assimilation system by $38 \%$ to $82 \%$, depending on the norm used to measure the error. Covariance inflation produced ensembles with analysis errors that were typically slightly less than the analysis errors from three-dimensional variational (3DVar) assimilation, but in order for the method to remain stable, the assimilations had to be continually restarted. An examination of the characteristics of the covariance inflation run showed that it developed perturbations that produced an inaccurate model of the background error covariances.

The most effective additive error parameterization used samples of model error from a time series of differences between T63 and T31 forecasts. Scaled samples of differences between model forecast states separated by $24 \mathrm{~h}$ were also tried, as well as scaled samples of the model state's anomaly from the model climatology. These latter two methods of generating additive error samples produced analyses that were less accurate than when using differences between T63 and T31 forecasts but more accurate than covariance inflation. Differences between T63 and T31 forecasts produced an additive error parameterization with a length scale very similar to the true model-error length scale, while the scaled 24-h forecast and deviations from climatology had progressively longer length scales, indicating that the correlation length scale of the additive errors is important.

This method of parameterizing model error does not use innovation statistics from the data assimilation, as proposed by Dee (1995), Dee and da Silva $(1998,1999)$ and tested in an ensemble filter by Mitchell and Houtekamer (2000). Use of innovation statistics has the concrete benefit in that model errors are estimated using independent information, the observations. The model-error parameters may also be adaptively tuned, increasing the magnitude of model error when the first guess is not a good fit to the observations. A drawback of the method is that if the structure of model errors is complex, it may not be possible to estimate a large number of parameters of a model-error covariance model using this approach. Also, the method depends on the observing network and an accurate characteriza- 
tion of the observation errors. Following the general suggestion of Mitchell and Houtekamer (2000, 430431), Houtekamer et al. (2005) assume that model errors resemble the more complex 3D-Var error covariances. In their ensemble assimilations, they generate scaled additive error samples that represent random draws consistent with these covariances. The scaling factor is adjusted to match time-averaged innovation statistics.

Of course, in our experiments we knew a priori what the model error was and thus could design a method of using forecasts from models at different resolutions to accurately simulate these model errors. In practice, the actual model deficiencies will not be very well known. They are likely to be an amalgamation of errors from many sources: errors in convective parameterizations, boundary layer parameterizations, radiation, cloud microphysics, land surface processes, parameter misestimation, model truncation, and so on. However, our approach is appealing at least for its simplicity; if one has a well-founded reason for believing that a particular parameterization is problematic, two models with different parameterizations could be run and the resulting differences used as samples of additive errors. Further, if additive errors come from many sources, there is no conceptual reason why multiple, independent additive errors could not be added to each ensemble member, one for each suspected type of model error. Perhaps an innovation statistics approach could be incorporated as well, adaptively changing the amount of model error added depending on the misfit of the first guess to the observations. Clearly, methods for parameterizing model error are important and deserving of much more exploration.

Acknowledgments. The lead author's participation was partially supported under National Science Foundation (NSF) Grants ATM-0130154, ATM-0112715, and ATM-0205612. Any opinions, findings, conclusions, or recommendations are those of the authors and do not necessarily represent the views of the NSF. The NOAA/FSL supercomputer resource hosted this experiment. Chris Snyder (NCAR) provided a helpful informal review of this manuscript, and Jeff Anderson (NCAR) is thanked for his consultations on ensemble assimilation issues. The authors thank two anonymous reviewers for their constructive criticism.

\section{REFERENCES}

Anderson, J. L., 2001: An ensemble adjustment Kalman filter for data assimilation. Mon. Wea. Rev., 129, 2884-2903.

_ 2003: A local least squares framework for ensemble filtering. Mon. Wea. Rev., 131, 634-642.
Buizza, R., M. Miller, and T. N. Palmer, 1999: Stochastic representation of model uncertainties in the ECMWF ensemble prediction system. Quart. J. Roy. Meteor. Soc., 125, 28872908.

Burgers, G., P. J. van Leeuwen, and G. Evensen, 1998: Analysis scheme in the ensemble Kalman filter. Mon. Wea. Rev., 126, 1719-1724.

Cohn, S. E., and D. P. Dee, 1988: Observability of discretized partial differential equations. SIAM J. Numer. Anal., 25, 585617.

Courtier, P., J.-N. Thépaut, and A. Hollingsworth, 1994: A strategy for operational implementation of 4D-Var, using an incremental approach. Quart. J. Roy. Meteor. Soc., 120, 13671387.

Dee, D. P., 1995: On-line estimation of error covariance parameters for atmospheric data assimilation. Mon. Wea. Rev., 123, $1128-1145$.

_ - and A. M. da Silva, 1998: Data assimilation in the presence of forecast bias. Quart. J. Roy. Meteor. Soc., 124, 269-295.

— , and — 1999: Maximum-likelihood estimation of forecast parameters for atmospheric data assimilation. Part I: Methodology. Mon. Wea. Rev., 127, 1822-1834.

_ and R. Todling, 2000: Data assimilation in the presence of forecast bias: The GEOS moisture analysis. Mon. Wea. Rev., 128, 3268-3282.

Etherton, B. J., and C. H. Bishop, 2004: Resilience of hybrid ensemble/3DVAR analysis schemes to model error and ensemble covariance error. Mon. Wea. Rev., 132, 1065-1080.

Evensen, G., 1994: Sequential data assimilation with a nonlinear quasigeostrophic model using Monte Carlo methods to forecast error statistics. J. Geophys. Res., 99, 10 143-10 162.

_ 2003: The ensemble Kalman filter: Theoretical formulation and practical implementation. Ocean Dyn., 53, 343-367.

_ _ and P. J. van Leeuwen, 1996: Assimilation of Geosat altimeter data for the Agulhas current using the ensemble Kalman filter with a quasigeostrophic model. Mon. Wea. Rev., 124, 85-96.

Gaspari, G., and S. E. Cohn, 1999: Construction of correlation functions in two and three dimensions. Quart. J. Roy. Meteor. Soc., 125, 723-757.

Gelb, A., Ed., 1974: Applied Optimal Estimation. MIT Press, 374 pp.

Hamill, T. M., and C. Snyder, 2000: A hybrid ensemble Kalman filter/3d-variational analysis scheme. Mon. Wea. Rev., 128, 2905-2919.

— , and —, 2002: Using improved background error covariances from an ensemble Kalman filter for adaptive observations. Mon. Wea. Rev., 130, 1552-1572.

__ J. S. Whitaker, and C. Snyder, 2001: Distance-dependent filtering of background error covariance estimates in an ensemble Kalman filter. Mon. Wea. Rev., 129, 2776-2790.

Houtekamer, P. L., and H. L. Mitchell, 1998: Data assimilation using an ensemble Kalman filter technique. Mon. Wea. Rev., 126, 796-811.

— atmospheric data assimilation. Mon. Wea. Rev., 129, 123-137.

,-- , G. Pellerin, M. Buehner, M. Charron, L. Spacek, and B. Hansen, 2005: Atmospheric data assimilation with an ensemble Kalman filter: Results with real observations. Mon. Wea. Rev., 133, 604-620.

Lawson, W. G., and J. A. Hansen, 2004: Implications of stochastic and deterministic filters as ensemble-based data assimilation 
methods in varying regimes of error growth. Mon. Wea. Rev., 132, 1966-1981.

Le Dimet, F.-X., and O. Talagrand, 1986: Variational algorithms for analysis and assimilation of meteorological observations: Theoretical aspects. Tellus, 38A, 97-110.

Legras, B., and R. Vautard, 1996: A guide to Lyapunov vectors. Proc. ECMWF Seminar on Predictability, Vol. 1, Reading, United Kimgdom, ECMWF, 143-156. [Available from ECMWF, Shinfield Park, Reading, Berkshire RG2 9AX, United Kingdom.]

Leith, C. E., and R. H. Kraichnan, 1972: Predictability of turbulent flows. J. Atmos. Sci., 29, 1041-1058.

Lorenc, A. C., 2003: The potential of the ensemble Kalman filter for NWP-A comparison with 4D-Var. Quart. J. Roy. Meteor. Soc., 129, 3183-3203.

Lorenz, E. N., 1969: The predictability of a flow which possesses many scales of motion. Tellus, 21, 289-307.

Maybeck, P. S., 1979: Stochastic Models, Estimation, and Control. Vol. 1, Academic Press, 423 pp.

Mitchell, H. L., and R. Daley, 1997: Discretization error and signal/error correlation in atmospheric data assimilation. Part I: All scales resolved. Tellus, 49A, 32-53.

— filter. Mon. Wea. Rev., 128, 416-433.

— - _ , and G. Pellerin, 2002: Ensemble size, balance, and model-error representation in an ensemble Kalman filter. Mon. Wea. Rev., 130, 2791-2808.

Palmer, T. N., 2001: A nonlinear dynamical perspective on model error: A proposal for non-local stochastic-dynamic parameterization in weather and climate prediction models. Quart. J. Roy. Meteor. Soc., 127, 279-304.

Parrish, D. F., and J. C. Derber, 1992: The National Meteorological Center's spectral statistical interpolation analysis system. Mon. Wea. Rev., 120, 1747-1763.

Rabier, F., J.-N. Thépaut, and P. Courtier, 1998: Extended assimilation and forecast experiments with a four-dimensional variational assimilation system. Quart. J. Roy. Meteor. Soc., 124, 1861-1887.

, H. Järvinen, E. Klinker, J.-F. Mahfouf, and A. Simmons, 2000: The ECMWF operational implementation of four- dimensional variational assimilation. I: Experimental results with simplified physics. Quart. J. Roy. Meteor. Soc., 126, $1143-1170$

Schubert, S. D., and M. Suarez, 1989: Dynamical predictability in a simple general circulation model: Average error growth. $J$. Atmos. Sci., 46, 353-370.

Simmons, A. J., and A. Hollingsworth, 2002: Some aspects of the improvement in skill of numerical weather prediction. Quart. J. Roy. Meteor. Soc., 128, 647-677.

Snyder, C., and T. M. Hamill, 2003: Leading Lyapunov vectors of a turbulent baroclinic jet in a quasigeostrophic model. $J$. Atmos. Sci., 60, 683-688.

- and F. Zhang, 2003: Assimilation of simulated Doppler radar observations with an ensemble Kalman filter. Mon. Wea. Rev., 131, 1663-1677.

Tippett, M. K., J. L. Anderson, C. H. Bishop, T. M. Hamill, and J. S. Whitaker, 2003: Ensemble square root filters. Mon. Wea. Rev., 131, 1485-1490.

Toth, Z., and E. Kalnay, 1997: Ensemble forecasting at NCEP and the breeding method. Mon. Wea. Rev., 125, 3297-3319.

Tribbia, J. J., and D. P. Baumhefner, 2004: Scale interactions and atmospheric predictability: An updated perspective. Mon. Wea. Rev., 132, 703-713.

Whitaker, J. S., and T. M. Hamill, 2002: Ensemble data assimilation without perturbed observations. Mon. Wea. Rev., 130, 1913-1924.

— , G. P. Compo, X. Wei, and T. M. Hamill, 2004: Reanalysis without radiosondes using ensemble data assimilation. Mon. Wea. Rev., 132, 1190-1200.

Zhang, F., C. Snyder, and J. Sun, 2004: Impacts of initial estimate and observation availability on convective-scale data assimilation with an ensemble Kalman filter. Mon. Wea. Rev., 132, $1238-1253$.

Zhang, S., and J. L. Anderson, 2003: Impact of spatially and temporally varying estimates of error covariance on assimilation in a simple atmospheric model. Tellus, 55A, 126-147.

Zou, X., A. Barcilon, I. M. Navon, J. Whitaker, and D. G. Cacuci, 1993: An adjoint sensitivity study of blocking in a two-layer isentropic model. Mon. Wea. Rev., 121, 2833-2857. 